\title{
Quantum fields in anti de Sitter spacetime and degrees of freedom in the bulk/boundary correspondence
}

\author{
Henrique Boschi-Filho*and Nelson R. F. Braga ${ }^{\dagger}$ \\ Instituto de Física, Universidade Federal do Rio de Janeiro \\ Caixa Postal 68528, 21945-970 Rio de Janeiro, RJ, Brazil
}

\begin{abstract}
The quantization of a scalar field in anti de Sitter spacetime using Poincaré coordinates is considered. We find a discrete spectrum that is consistent with a possible mapping between bulk and boundary quantum states.
\end{abstract}

*e-mail: boschi @ if.ufrj.br

$\dagger$ †e-mail: braga @ if.ufrj.br 
The holographic principle asserts that the degrees of freedom of a quantum system with gravity can be represented by a theory on the boundary [1, 2, 3]. The presence of gravity makes it possible to define a mapping between theories defined in manifolds of different dimensionality. One interesting realization of the holographic principle can be done in a space of constant negative curvature, the anti de Sitter ( $A d S)$ spacetime. Such a realization was proposed by Maldacena in the form of a conjecture [4 on the equivalence (or duality) of the large $N$ limit of $S U(N)$ superconformal field theories in $n$ dimensions and supergravity on anti de Sitter spacetime in $n+1$ dimensions $(A d S / C F T$ correspondence). Then, using Poincaré coordinates in the AdS bulk, Gubser, Klebanov and Polyakov [5] and Witten [6] found prescriptions for relating theories that live in the bulk and on the boundary, where the AdS solutions play the role of classical sources for the boundary field correlators.

Despite the fact that field quantization in AdS in terms of global coordinates has been known for a long time [7, 8], the corresponding formulation in Poincaré coordinates and thus a comprehensive picture of holography in terms of bulk quantum fields is still lacking. The aim of the present letter is to investigate a quantum theory for a scalar field in the AdS bulk in terms of Poincaré coordinates. We will see that the dimensionality of the phase space is such that a mapping between this theory and states on the boundary is possible. This conclusion essentially depends on the fact that the AdS space in Poincaré coordinates should be compactified, as it happens in the usual global coordinates, in order to include appropriate boundary conditions at infinity and find a consistent quantization. Although the Poincaré coordinates extend to infinity we will need to introduce a finite radius (cutoff) $R$ corresponding to the fact that we can not represent the whole compactified AdS space used in the AdS/CFT correspondence (including the infinity) into just one single set of Poincaré coordinates. Naturally, we can take $R$ large enough to describe as much of the entire AdS space as we want. We are going to see in the following that this result is in agreement with the counting of degrees of freedom in the bulk/boundary correspondence.

The anti-de Sitter spacetime of $n+1$ dimensions can be represented as the hyperboloid ( $\Lambda=$ constant $)$ 


$$
X_{0}^{2}+X_{n+1}^{2}-\sum_{i=1}^{n} X_{i}^{2}=\Lambda^{2}
$$

in a flat $n+2$ dimensional space with metric

$$
d s^{2}=-d X_{0}^{2}-d X_{n+1}^{2}+\sum_{i=1}^{n} d X_{i}^{2} .
$$

The so called global coordinates $\rho, \tau, \Omega_{i}$ for $A d S_{n+1}$ can be defined by [9, 10]

$$
\begin{aligned}
X_{0} & =\Lambda \sec \rho \cos \tau \\
X_{i} & =\Lambda \tan \rho \Omega_{i} \quad\left(\sum_{i=1}^{n} \Omega_{i}^{2}=1\right) \\
X_{n+1} & =\Lambda \sec \rho \sin \tau,
\end{aligned}
$$

with ranges $0 \leq \rho<\pi / 2$ and $0 \leq \tau<2 \pi$.

Poincaré coordinates $z, \vec{x}, t$ can be introduced by

$$
\begin{aligned}
X_{0} & =\frac{1}{2 z}\left(z^{2}+\Lambda^{2}+\vec{x}^{2}-t^{2}\right) \\
X_{i} & =\frac{\Lambda x^{i}}{z} \\
X_{n} & =-\frac{1}{2 z}\left(z^{2}-\Lambda^{2}+\vec{x}^{2}-t^{2}\right) \\
X_{n+1} & =\frac{\Lambda t}{z}
\end{aligned}
$$

where $\vec{x}$ has $n-1$ components and $0 \leq z<\infty$. In this case the $A d S_{n+1}$ measure with Lorentzian signature reads

$$
d s^{2}=\frac{\Lambda^{2}}{(z)^{2}}\left(d z^{2}+(d \vec{x})^{2}-d t^{2}\right) .
$$

Then the $A d S$ boundary described by usual Minkowski coordinates $\vec{x}, t$ corresponds to the region $z=0$ plus a "point" at infinity $(z \rightarrow \infty)$.

In order to gain some insight into the form of the spectrum associated with quantum fields to be defined in the AdS/CFT framework, let us discuss an essential point of the correspondence: the mapping between the degrees of freedom of the bulk volume and those of the boundary hypersurface. The metric is singular at $z=0$, so the prescriptions [5, 6] for calculating field correlators should be first taken at some small $z$ that then goes 
to zero [11, 12, 10, 13]. In the same way, we will consider the boundary to be at some small $z=\delta$.

So, let us consider at $z=\delta$ a hypersurface of area $\Delta A$ corresponding to variations $\Delta x^{1} \ldots \Delta x^{n-1}$ in the space coordinates:

$$
\Delta A=\left(\frac{\Lambda}{\delta}\right)^{n-1} \Delta x^{1} \ldots \Delta x^{n-1}
$$

and calculate the volume generated by this surface moving $z$ from $\delta$ to $\infty$, finding

$$
\Delta V=\Lambda \frac{\Delta A}{n-1}
$$

This is the expected result that the volume is proportional to the area in the bulk/boundary correspondence for a fixed $\Lambda$. In order to count the degrees of freedom we can split $\Delta V$ in $\ell$ pieces of equal volume corresponding to cells whose boundaries are hypersurfaces located at

$$
z_{j}=\frac{\delta}{\sqrt[n-1]{1-j / \ell}},
$$

with $j=1, \ldots, \ell-1$. Note that the last cell extends to infinity. These volume cells can be mapped into the area $\Delta A$ by dividing it also in $\ell$ parts. This way, one finds a one to one mapping between degrees of freedom of bulk and boundary. This analysis shows us that despite the fact that the variable $z$ has an infinite range, the volume, and thus the associated degrees of freedom, corresponding to a finite surface, are finite. One could, in a simplified way, think of the system as being "in a box" in terms of degrees of freedom, with respect to $z$. We could have changed $z$ to a variable that measures the volume, say $\zeta=1 / \delta^{n-1}-1 / z^{n-1}$, to explicitly find this compact role of the radial coordinate $z$ but this would not be better than just going to global coordinates, eqs.(3)). However we want to see from the point of view of the Poincaré coordinates, where the AdS/CFT correspondence takes its more natural form, how does this compactified character of the radial coordinate manifests itself.

Then, let us consider a massive scalar field $\phi$ in the $A d S_{n+1}$ spacetime described by Poincaré coordinates with action

$$
I[\phi]=\frac{1}{2} \int d^{n+1} x \sqrt{g}\left(\partial_{\mu} \phi \partial^{\mu} \phi+m^{2} \phi^{2}\right)
$$


where we take $x^{0} \equiv z, x^{n+1} \equiv t, \sqrt{g}=\left(x^{0}\right)^{-n-1}$ and $\mu=0,1, \ldots, n+1$.

The classical equation of motion reads

$$
\left(\nabla_{\mu} \nabla^{\mu}-m^{2}\right) \phi=\frac{1}{\sqrt{g}} \partial_{\mu}\left(\sqrt{g} \partial^{\mu} \phi\right)-m^{2} \phi=0
$$

and the solutions can be found [14, 15] in terms of Bessel functions using the ansatz $\phi=$ $e^{-i \omega t+i \vec{k} \cdot \vec{x}} z^{n / 2} \chi(z)$. Taking $\omega^{2}>\vec{k}^{2}$ and defining $u=\sqrt{\omega^{2}-\vec{k}^{2}}$ and $\nu=\frac{1}{2} \sqrt{n^{2}+4 m^{2}}$, we have two independent solutions

$$
\Phi^{ \pm}=e^{-i \omega t+i \vec{k} \cdot \vec{x}} z^{n / 2} J_{ \pm \nu}(u z)
$$

if $\nu$ is not integer. If $\nu$ is integer one can take $\Phi^{+}$and

$$
\Phi^{-}=e^{-i \omega t+i \vec{k} \cdot \vec{x}} z^{n / 2} Y_{\nu}(u z)
$$

as independent solutions.

On the other hand, if $\vec{k}^{2}>\omega^{2}$ the solution is

$$
\bar{\Phi}=e^{-i \omega t+i \vec{k} \cdot \vec{x}} z^{n / 2} K_{\nu}(q z)
$$

where $q=\sqrt{\vec{k}^{2}-\omega^{2}}$ (the second solution in this case is proportional to $I_{\nu}(q z)$ which is divergent as $z \rightarrow \infty)$.

As discussed in refs. 14, 15, $\Phi^{+}$are the only normalizable solutions in the range $0<z<\infty$. They are thus the natural candidates for the role of quantum fields, if we want to be able to take the limit of $\delta \rightarrow 0$ at the end. One could then naively think of just adding all possible solutions $\Phi^{+}$and thus building up a quantum field like

$$
\int d u d^{n-1} k f(\vec{k}, u) \Phi^{+}(\vec{k}, u)+\text { c.c. . }
$$

However, from our previous analysis of degrees of freedom, we expect to find a discrete spectrum associated with the radial coordinate $z$. Such a discretization would be in accordance with the results coming from the quantization in global coordinates [7, 8, 14, 15].

One can understand why this discretization also takes place in Poincare coordinates by considering a simpler situation: the stereographic mapping of the surface of a sphere on 
a plane. One can map the points of a sphere on a plane plus a point at infinity. However looking at the sphere one sees that this compact manifold has discrete sets of eigenfunctions but looking at the plane how can we realize that the spectrum of eigenfunctions in the radial direction would be discrete? In close analogy with the case of finite volumes $\Delta V$ for $z \rightarrow \infty$ in AdS discussed above, here in the case of the sphere if we calculate the area of the plane taking the metric induced by the sphere into account we would find a finite value (equal to the area of the sphere). So, the radial coordinate on the plane looks also like a "compact" one (in the sense of degrees of freedom or whatever we associate with area cells) in the same way as the $z$ coordinate of AdS. The extra point at infinity corresponds to the fact that we should impose the condition that going to infinity in any direction would led to the same point. This condition would mean that either the functions on the plane have no angular dependence or they vanish as the radial coordinate tend to zero. These conditions would not yet lead to a discrete spectrum (in the radial direction). This problem is simply related to the fact that the point at infinity, which has zero measure, is not represented on the plane. If instead of using just one plane, we project the sphere on two different planes we would be able to represent all the points of the sphere. We could even choose one of the mappings to cover "as much of the surface of the sphere" as we want. As long as we map it into two disks of finite radius (with an appropriate matching boundary condition), one would then clearly see that the spectrum of eigenfunctions is discrete.

Now coming back to the AdS case, a consistent quantization in this space in global coordinates [7, 8] requires the introduction of boundary conditions at the surface corresponding to $\rho=\pi / 2$ in order to have a well defined Cauchy problem. So, one must consider a compactified AdS including $\rho=\pi / 2$ in order to find a consistent theory.

The limit $z \rightarrow \infty$ in Poincaré coordinates (4) corresponds to a point that in global coordinates sits in the hypersurface $\rho=\pi / 2$. Thus, this hypersurface is not completely represented in just one set of Poincaré coordinates. In the same way as in the case of the sphere, we can solve this problem mapping the compactified AdS in two sets of Poincaré coordinates. We can simply stop at $z=R$ in one set and map the rest, including the point at infinity, in a second set. We can take $R$ arbitrarily large so that we can map as much of the compactified AdS spacetime as we want in just one set. 
In this region $0 \leq z \leq R$ we can introduce as quantum fields

$$
\Phi(z, \vec{x}, t)=\sum_{p=1}^{\infty} \int \frac{d^{n-1} k}{(2 \pi)^{n-1}} \frac{z^{n / 2} J_{\nu}\left(u_{p} z\right)}{R w_{p}(\vec{k}) J_{\nu+1}\left(u_{p} R\right)}\left\{a_{p}(\vec{k}) e^{-i w_{p}(\vec{k}) t+i \vec{k} \cdot \vec{x}}+c . c .\right\}
$$

where $w_{p}(\vec{k})=\sqrt{u_{p}^{2}+\vec{k}^{2}}$ and $u_{p}$ are such that $J_{\nu}\left(u_{p} R\right)=0$.

Imposing that the operators $a, a^{\dagger}$ satisfy the commutation relations

$$
\begin{aligned}
& {\left[a_{p}(\vec{k}), a_{p^{\prime}}^{\dagger}\left(\vec{k}^{\prime}\right)\right]=2(2 \pi)^{n-1} w_{p}(\vec{k}) \delta_{p p^{\prime}} \delta^{n-1}\left(\vec{k}-\vec{k}^{\prime}\right)} \\
& {\left[a_{p}(\vec{k}), a_{p^{\prime}}\left(\vec{k}^{\prime}\right)\right]=\left[a_{p}^{\dagger}(\vec{k}), a_{p^{\prime}}^{\dagger}\left(\vec{k}^{\prime}\right)\right]=0}
\end{aligned}
$$

we find, for example, for the equal time commutator of field and time derivative

$$
\left[\Phi(z, \vec{x}, t), \frac{\partial \Phi}{\partial t}\left(z^{\prime}, \vec{x}^{\prime}, t\right)\right]=i z^{n-1} \delta\left(z-z^{\prime}\right) \delta\left(\vec{x}-\vec{x}^{\prime}\right) .
$$

Now considering again the field (15) we realize that the discretization of the spectrum makes it possible to map the phase space $u_{p}, \vec{k}$ into the momentum space of a field theory defined on the boundary, in the same way as we can map an infinite but enumerable set of lines into just one line.

Taking, for simplicity, $A d S_{3}$ where $\vec{k}$ has just one component, the phase space in the bulk would be an enumerable set of lines each one corresponding to the continuous values of $-\infty<k<\infty$ and one fixed value of $p$. One can map these lines into just one line, corresponding to some momentum, say $\kappa_{b}$ on the boundary, by dividing the line of $\kappa_{b}$ into segments of finite size. This kind of mapping would not be possible if the spectrum were not discrete, as one can not define a one to one mapping between a plane and a line. So, the discretization of the spectrum is a necessary ingredient for the holographic mapping to hold.

In conclusion, we have obtained a quantum scalar field in the AdS bulk that exhibits a discrete spectrum associated with the radial Poincare coordinate. This result was obtained taking into account the compactification of AdS that in Poincare coordinates corresponds to adding a point at infinity. This discretization is in agreement with the counting of degrees of freedom suggested by the holographic principle. However it is in contrast to the continuous spectrum found in ref. [16]. 


\section{Acknowledgments}

The authors were partially supported by CNPq, FINEP and FUJB - Brazilian research agencies. We also thank Mauricio Calvão, Juan Mignaco, Cassio Sigaud and Arvind Vaidya for interesting discussions.

\section{References}

[1] G. 't Hooft, "Dimensional reduction in quantum gravity" in Salam Festschrifft, eds. A. Aly, J. Ellis and S. Randjbar-Daemi, World Scientific, Singapore, 1993, grqc/9310026.

[2] L. Susskind, J. Math. Phys. 36 (1995) 6377.

[3] L. Susskind and E. Witten, "The holographic bound in anti-de Sitter space", SUITP-98-39, IASSNS-HEP-98-44, hep-th 9805114.

[4] J. Maldacena, Adv. Theor. Math. Phys. 2 (1998) 231.

[5] S. S. Gubser , I.R. Klebanov and A.M. Polyakov, Phys. Lett. B428 (1998) 105.

[6] E. Witten, Adv. Theor. Math. Phys. 2 (1998) 253.

[7] S. J. Avis, C. J. Isham and D. Storey, Phys. Rev. D18 (1978) 3565.

[8] P. Breitenlohner and D. Z. Freedman, Phys. Lett. B115(1982) 197; Ann. Phys. 144 (1982) 249.

[9] O. Aharony, S.S. Gubser, J. Maldacena, H. Ooguri and Y. Oz, Phys. Rept 323 (2000) 183.

[10] J. L. Petersen, Int. J. Mod. Phys. A14 (1999) 3597.

[11] W. Mueck and K. S. Viswanathan, Phys. Rev. D58(1998)041901.

[12] D. Z. Freedman, S. D. Mathur, A. Matusis and L. Rastelli, Nucl. Phys. B546 (1999) 96. 
[13] H. Boschi-Filho and N. R. F. Braga, Phys. Lett. B 471 (1999) 162.

[14] V. Balasubramanian, P. Kraus and A. Lawrence, Phys. Rev. D59 (1999) 046003;

[15] V. Balasubramanian, P. Kraus, A. Lawrence and S. P. Trivedi, Phys. Rev. D59 (1999) 1046021;

[16] M. M. Caldarelli, Nucl. Phys. B549 (1999) 499. 